\title{
Einleitung und Bearbeitungshinweise
}

Dieses Übungs- und Arbeitsbuch soll zur anwendungsorientierten Vertiefung der mittels des Lehrbuches „Angewandtes Unternehmenscontrolling - Operative Systeme der Planung, Entscheidung und Kontrolle“ (Behrens, Feuerlohn 2018) erworbenen Kenntnisse und Fähigkeiten beitragen. Es ist konzeptionell und gliederungsseitig mit der ersten Auflage des Lehrbuches vollständig kompatibel.

Die Erarbeitung und die Bereitstellung entscheidungsrelevanter Informationen für das Treffen kurzfristig wirksamer Entscheidungen als operative Kernaufgaben des Controllings stehen auch im Übungs- und Arbeitsbuch im Mittelpunkt der Übungen und Anwendungsbeispiele. Als Ausgangspunkt ist - wie im Lehrbuch - eine entscheidungsorientierte Ausrichtung des Controllings gewählt.

\section{Allgemeine Hinweise}

Das Arbeits- und Übungsbuch gliedert sich in zwei Teile:

- Die Übungsaufgaben im Teil I des Übungs- und Arbeitsbuches dienen zur Einübung der Informationssysteme der finanz- und vor allem der kostenorientierten Controllingausrichtungen. Die Übungsaufgaben beziehen sich somit überwiegend auf den Teil A und ergänzend auch auf den Teil F des Lehrbuches; sie sind mit überwiegend eindeutigen Lösungen ausgestattet. Mit diesen Übungsaufgaben soll das notwendige Fundament für das Verständnis des operativen Controllinginstrumentariums erarbeitet werden. Aufgrund ihrer herausragenden Bedeutung für das operative Controlling stehen hier die Voll- und Teilkostenrechnungssysteme im Zentrum der Übungen.

- Die themenübergreifenden Fallstudien im Teil II des Übungs- und Arbeitsbuches stellen auf die fallbezogene Entwicklung von Plan- und Budgetvorgaben, von Kalkulationen und Erfolgsrechnungen, von Abweichungsanalysen und Entscheidungsvorlagen, sowie von Koordinationsvorschlägen ab. Im Mittelpunkt stehen hier - wie im Lehrbuch - die Budgetierung, das Berichtswesen, die Koordination dezentraler Unternehmenseinheiten, die kurzfristig wirksamen Produktionsprogramm- und Preissteuerungen, sowie die jeweils resultierenden Verhaltenswirkungen. Da die fallbezogene, exemplarische Umsetzung des operativen Controllings stellenweise durchaus verschiedene Lösungswege und Ergebnisse zulässt, sind die Fallstudien stellenweise mit exemplarischen Lösungshinweisen ausgestattet. Die jeweils geforderten kritischen Reflexionen der erarbeiteten Lösungen zu den Fallstudien stellen auf die Vielfalt der Lösungswege und -vorschläge $\mathrm{ab}$. 
Um einen themenzentrierten Zugang zu den Übungsaufgaben und Fallstudien zu gewähren, ist dem Übungs- und Arbeitsbuch ein Themenregister beigefügt. Dieses Register weist die zentralen Rechnungssysteme des operativen Controllings mit ihren Anwendungen in den Übungsaufgaben und Fallstudien aus.

Im Rahmen der Bearbeitung der Fallstudien und Aufgaben verstehen Sie sich bitte als unternehmensinternes Beratungszentrum, das für die Entscheidungsträger innerhalb des Unternehmens Entscheidungsvorlagen zu erarbeiten und damit Entscheidungen vorzubereiten hat - damit aber auch Verhaltenswirkungen bei den Entscheidungsträgern erzeugt bzw. erzeugen kann.

\section{Bearbeitungshinweise}

Für die Bearbeitung der Übungen und Fallstudien sind Grundlagenkenntnisse der Allgemeinen Betriebswirtschaftslehre und des betriebswirtschaftlichen Rechnungswesens von großem Vorteil. Dies betrifft insbesondere die Grundlagen der doppelten Buchführung in Konten und die elementaren Grundlagen der Kosten- und Leistungsrechnung. Daneben sind auch Grundlagenkenntnisse der Organisationslehre sowie der Unternehmens- und Personalführung hilfreich. Zur Rekonstruktion dieser grundlegenden Sachverhalte seien hier empfohlen:

- Behrens, Reinhard/Feuerlohn, Bernd (2018): Angewandtes Unternehmenscontrolling. Operative Systeme der Planung, Kontrolle und Entscheidung [Lehrbuchteile A und F], Berlin et al.: De Gruyter Oldenbourg.

- Jung, Hans (2016): Allgemeine Betriebswirtschaftslehre, 13., aktualisierte Auflage, Berlin et al.: De Gruyter Oldenbourg.

- Jung, Hans (2012): Arbeits- und Übungsbuch Allgemeine Betriebswirtschaftslehre, 4. Auflage, München: Oldenbourg.

- Jung, Hans (2014a): Controlling, 4., aktualisierte Auflage, München: Oldenbourg.

- Jung, Hans (2014b): Arbeitsbuch Controlling. Aufgaben und Testfragen mit Lösungen zur Klausurvorbereitung, 2., korrigierte und aktualisierte Auflage, München: Oldenbourg.

- Eisele, Wolfgang/Knobloch, Alois Paul (2018): Technik des betrieblichen Rechnungswesens: Buchführung und Bilanzierung, Kosten- und Leistungsrechnung, Sonderbilanzen, 9., vollständig überarbeitete und erweiterte Auflage, München: Vahlen.

- Fischbach, Sven (2017): Grundlagen der Kostenrechnung, 7. Auflage, München: Vahlen.

- Haberstock, Lothar/Breithecker, Volker (2008): Kostenrechnung I. Einführung mit Fragen, Aufgaben, einer Fallstudie und Lösungen, 13., neu bearbeitete Auflage, Berlin: ESV.

- Kalenberg, Frank (2013): Kostenrechnung: Grundlagen und Anwendungen - mit Übungen und Lösungen, 3. Auflage, München: Oldenbourg. 
- Thommen, Jean-Paul/Achleitner, Ann-Kristin/Gilbert, Dirk Ulrich/Hachmeister, Dirk/Kaiser, Gernot (2017): Allgemeine Betriebswirtschaftslehre. Umfassende Einführung aus managementorientierter Sicht, 8., vollständig überarbeitete Auflage, Wiesbaden: Springer Gabler.

- Thommen, Jean-Paul/Achleitner, Ann-Kristin/Gilbert, Dirk Ulrich/Hachmeister, Dirk/Jarchow, Svenja/Kaiser, Gernot (2018): Allgemeine Betriebswirtschaftslehre Arbeitsbuch - Repetitionsfragen, Aufgaben, Lösungen, 8., vollständig überarbeitete Auflage, Wiesbaden: Springer Gabler.

- Wöhe, Günter/Döring, Ulrich/Brösel, Gerrit (2016a): Einführung in die Allgemeine Betriebswirtschaftslehre, 26., überarbeitete und aktualisierte Auflage, München: Vahlen.

- Wöhe, Günter/Döring, Ulrich/Brösel, Gerrit (2016b): Übungsbuch zur Einführung in die Allgemeine Betriebswirtschaftslehre, 15., überarbeitete und aktualisierte Auflage, München: Vahlen.

Auch wenn die Übungsaufgaben und die Fallstudien im Übungs- und Arbeitsbuch mit Lösungen bzw. Lösungshinweisen ausgestattet sind, sollte im Interesse der Erreichung der Lernziele zunächst eine Bearbeitung ohne vorherige Sichtung der Lösungen bzw. Lösungshinweise erfolgen. Die Lösungen bzw. Lösungshinweise sollen vor allem der Selbstkontrolle dienen, und erst im Rahmen einer Überprüfung der selbst entwickelten Lösungsvorschläge eingesetzt werden. Im Kontext der mathematischen Aufgabenbearbeitung sind gegebenenfalls erforderliche Rundungen grundsätzlich auf die zweite Nachkommastelle vorzunehmen!

Für die Bearbeitung der themenübergreifenden Fallstudien gewinnt der Begriff der Komplexität in betriebswirtschaftlicher Perspektive besondere Bedeutung, da die verschiedenen Rechnungssysteme, die zur Erarbeitung von Lösungsvorschlägen und Entscheidungsvorlagen eingesetzt werden, letztlich Instrumente zur Reduktion der fallbezogenen Komplexitäten darstellen. Erst durch den Einsatz der komplexitätsreduzierenden Rechnungssysteme werden eine Bewältigung der in den Fallstudien vorliegenden vernetzten Problemsituationen und der mit ihnen verbundenen Optimierungsherausforderungen möglich. Da die somit grundsätzlich notwendigen Komplexitätsreduktionen zugleich Abstraktionen von der betriebswirtschaftlichen Realität bewirken, stehen die eingesetzten Rechnungssysteme in der potenziellen Gefahr, zur Realitätsferne der Lösungsvorschläge beizutragen, oder gar zur gezielten Gestaltung der resultierenden Entscheidungsvorlagen eingesetzt zu werden. Insofern bedarf es aus betriebswirtschaftlicher, aber auch aus übergeordneter wissenschaftlicher Perspektive immer einer Reflexion der Folgewirkungen der herbeigeführten Komplexitätsreduktionen.

Für die Bearbeitung der Übungsaufgaben und der Fallstudien wünschen wir Ihnen maximalen Erfolg und durchaus auch ein wenig Freude. Neben den Lösungen bzw. den Lösungshinweisen und dem Themenregister bieten die fallbezogenen Quellenhinweise, die orientierenden Grundlegungen $\mathrm{zu}$ den Begriffen der Komplexität 
und der Komplexitätsreduktion, sowie das fallstudienbezogene Glossar des Übungsund Arbeitsbuches weitere Wege zur Aufgaben- und Fallbearbeitung und zur zielorientierten Erarbeitung und Vertiefung der Fachinhalte.

Bei der Erstellung des Manuskriptes für das Übungs- und Arbeitsbuch durften wir uns auf geduldige Hilfestellung verschiedener Personen verlassen. Unser besonderer Dank gilt erneut den Mitarbeiterinnen und Mitarbeitern der Hochschulbibliothek Nordhausen für die zeitnahe Materialbeschaffung und -bereitstellung. Dem Senior Acquisitions Editor des Verlages, Herrn Dr. Stefan Giesen, sind wir für die konstruktive Zusammenarbeit ebenfalls sehr verbunden.

Auch bei mehrfachem Korrekturlesen steht leider eine vollständige Erfassung und Elimination aller Fehler nicht zu erwarten. Aus diesem Grunde sind wir für entsprechende Rückmeldungen (an: unternehmenscontrolling@hs-nordhausen.de) recht dankbar! 